Die Carbolsäure hewälrte sich in vielen Fällen. Doch gelang es nicht in jedem, mit dersellen die Säuerung des lirines herbeizuführen. llıre Einwirkung ist auch hier wesentlich die eines Antisepticums. weshall, sie bei starker II arngïhrung und besonders bei deu diphtheritischen Processen der Hlase, da aler in concentrirteren Lösungen, (cl. oben) eimpfohlen werden kann. Auf den Selleinhlautkatarrh selber haben dic gewöhnlich gehrauchten Jïsungen wohl nur einen geringen, vielleiclt nur indirecten Einfluss; mehr dagegen leisten sie in Fällen wo die Schleimhaut reichlich Eiterkörperchen producirt. Da nimmt unter dem Gehrauch der Carbolsäure die Ejtermenge oft rasch al. Stark schleimige, zälıe Sedinente werlen von den schwachen Lüsungen, wie ich anich experimeutell überzeugt hale, kaun merkbar verändert. laraus scleciut es sich zu erklären, weshalb man in den Fällen, wo solche Sedimente vorhanden sind, mit ihr nicht zum Zicle kommt.

Die Salicylsäure ergalb bei äusserer Application weil bessere Resullate, wie hei interner. Sic wirkt nicht bloss als ein gutes Antisepticum, sondern wandelt besonders leicht vorwiegend eiterige Sedimente in eine feinkärnige Emulsion um, welche sich sehr vollständig aus der Blase:

\section{Ueber die Localbehandlung des chronischen Blasenkatarrhs.} Von

Dr. M. Schtiller,

Docent der Universităt Greifswald und Assistent der chirurgischen Kklinik.

(Schluss aus No. 8.)

Was endlich die speciellere Wirkung und diflerentielle ludication der gewählten Mittel aulangt, so sollen sie, wie schon oben hervorgehoben, wesentlich zwei Aufgaben exfillen, nämlich theils a uf the $Z$ ersetzung des Harnes, theils auf die katarrlalisch afficirte Blasenschleimhaut einwirken. In Rücksicht hierauf kann man sie in antiseptische, adstringirende, resolvirende Mittel eintheilen, wenn auch in Wirklichlieit eine solche Classification wenig practischen Werth hat, da die meisten der Mittel gemischte Wirkungen haben und besonders die antiseptischen in nicht geringerem Grade auch adstringirend wirken. -

Neben dem Werthe für die Erfíllung der beiden Indicationen muss jedoch für dic Wahl der einzelnen Mitlel nicht weniger eine ander Eigensehiaft bestimmend sein, durch welche die Eutleerumg der Blase in allen Fällen die Hauptaufgabe - erheblich gefirdert unl erleichter werden kann. Es nüssen nämlich die angewandten Mittel bei ilırer chemischen Einwirkung auf den Blaseniubalt denselben zugleich in eine solche mechanische Form bringen, dass seine Entleerung a us der Blase leicht und collkommen von statten geht. Ja die Erfüllung dieser wichtigsten Indication bei der Behandlung des clıronischen Blasenkatarrlıs selır häufig durch ein den Harn beigemengtes dickes Sediment von Harnsalzen, Schleim, Eiterkörperchen erhel)lich erschwert wird, so ist es nothwendig, dass vorwiegend solche Lösungen zu Spülflüssigkeiten benutzt werlen, welche tli e grallertigen, zähflüssigen, dem Blasengrunde anhaftenden Niederschläre entweder zu lösen oder doch zu verflüssigen im Stande silld, oder welche sie in eine feinkörnige Emulsion umzuwandeln vermögen. Diese Aufgabe ist in der That mindestens ebenso wesentliclı, wie z. B. die Einfülırung antiseptischer Flïssigkeiten. Denn nur auf diese Weise wird es gelingen, die Blase wirklich vollkommen zu entleeren und von dem Reize dieser leicht zersetzlichen, immer wieder von Neuen den Katarrh und die Harnzersetzung hervorrufenden Massen zu befreien. Die Desinfection vermag fïr sich allein diese gallertigen Niederschläge nicht unschädlich zu machen, da die desinficirenden Mittel gar nicht in der Concentration angewandt werden liönnen, welche nothwendigr wäre, un die Zersetzung und Zersetzungstähigkeit der Niederschläge aufzuhebeu. Die Einwirkung der antiseptischen Lösungen ist gleichwohl keineswegs zu verkennen; sie kommt aljer augenscheinlich weit besser und erst wesentlich dann zur Geltung, wenu die stagnirenden Niederschläge ordentlich entleert werden. In dieser Beziehung verlıält sich die Blase nichl anders wie eime Abscesshöhle. Wie wir diese trotz aller möglichen desinficirenden Einspritzungen nicht zur Ausheilung bringen können, wenn wir nicht zugleich für einen genügenden Abfluss der Secrete sorgen, so bleibt auch trotz desinfiicirender Ausspülungen der deletäre Einfluss der Sedimente auf Blasenschleimhaut und Harn fortbestehen, wenn nicht zugleich durch jene das Sediment selber stets mit entfernt wird.

Von den von uns angewandten Mitteln genügen die meisten nur der einen oder anderen Anforderung. Um speciell ilıre Einwirkung auf das gallertig-schleimige Sediment gewisser chronischer Blasenkatarrhe kennen zu lernen habe ich sie jetles für sicl mit Portionen von IIarnniederschlägen in Reagensgläsem gemisclıt und die etwaigen Veränderungen theils makroskopisch, theils milkroskopisch geprüf. Die Ergebnisse dieser Untersuchung, welche in gutem Einklange mit unseren practischen Erfahrungen über den Wirkıngsmodus der eimzelnen Mittel stelıen, sind auch bei den gleich folgenden Angaben hieriber nit verwerthet worden. entleeren lässt. Die Eiterkörperchen verlieren unter dem Einflusse verdünnter Salicylsäurelïsungen dic Fähigkeit aneinander zu haften. Auch lässt sich ilar eine sehr dentliche Einwirkung auf den Katarrh der Schleimhaut sellser nicht absprechen. Unater ihrer Application verminderte sich in den meisten Fälen selır hahl, augenscheinlich noch rascher wie unter der Carboleinwirkung, die Eiteralssonderung und die Abstossung vou Epithelien. Gegeniiber den lickschleimigen Sedimenten zeigte sie sich jedoch auch in einigen Fällen machtlos. Gleichwohl möchte ich sie zu allgemeinerem Gelurauche empfehlen. Man wird mit ilir allein in vicleu Fällen vollkommen ausreichen. Sie vereinigt in sich vielleicht unter allen von uns angewandten Mitleln am besten und vollständhgsten fast alle zur Erfiillung der verschiedenen oben hervorgehobenen Indicationen erforderliclien Bedingungen, indem sie sowohl antiseptisch auf den Blaseninhalt und adstringirend auf die Blasenschleimhaut, wie emulgirend wenigstens auf die eiterigen Sedimente eimwirkt. - Verhuhlen darf ich hier nicht, dass bei ihrem Gebranch zuweilen mässige Resorptionserscheinungen bemerkbar werden; - aber dieselben sind practisch durchatus bedeutungslos und ungefährlich; und jedenfalls keineswegs derart, uu von der localen Anwendung der Salicylsäure bei Blasenkatatrhen ilbzuhalten.

Vom kali hypermanganicum, welches wir nur einige Male in Anwendung gezogen habeu, kann ich nur wenig aussagen. In den betrefienten Fällen wirkte es als ein recht gutes Antisepticum. Auch wurde der Katarrl und das Sediment geringer. In der Regel werden nur schwadhe Lisungen vertragen, da stärkere leicht Schmerzen machen.

liein Mittel eignet sich so vortrefflich zur Lösung schleimig eitriger und dickzälıer Sedimente vie clas Chlornatrium. Es hat uns in allen den Fällen, in welchen wir es angewenclet lıaben, niemals im Stich gelassen. Dasselbe wurde von mir zunächst aus theoretischen Erwägungen versuchit. Die schleimigen Massen des Harnes beim chronischen Blasenkatarrh bestelıen bekanntlich wesentlich aus gequollenen Epithelièn und Eiterköperchen, von welchen besonders die letzteren grosse Mengeu Myosin (nicht Mucin!) enthalten. Dieses tritt nur zum kleiusten Theile bei längerer Harustauung in den Harn über, bewirkt dagegen wahrscheinlich durch Imbibition das Quellen und zälıe Aneinanderhaften der Eiterkörperchen und giebt so die Ursache zur Umıwandlung derselben in die stark klebrigen, am Blasengrunde anhaftenden, schwer entfernbaren dicken Niederschläge, denen man so oft bei chronischen Blasenkatarrhen begegnet. Da nun notorisch ') Myosin von Chlornalrium lösungen in eine lösliche Form übergefülırt wirl, so hoffe ich, auf diese Weise durch Chlornatriumausspiilungen die Blase von dem zälıen Sedimente zu befreien. Der Erfolg bestätigte diese theoretischın Vorstellungen vollkomınen. Eb enso wie im Reagensglase werden in der Blase die cohärenten Massen in eine selur gleichmässige milchige Emulsion gebracht, welche leicht abfliesst, so dass man annehmen kanm, dass in der That nach der jedesmaligen Ausspiulung nichts mehr am Grunde der Blase haftet. Auf diese vollständige Entlecrung der Blase darf nan auch, wie iclı glaube, das nach der Chlornatriumapplication meist rasch eintretende Sauerwerden les Harnes beziehen, wiewohl hierauf auch die Verminderung des Blasenkatarrhs selber von einigem Einfuss sein wird.

Die Einspritzungen von Argentum nitricum - und Chlorzinklösungen geschehen vortheilhaft besonders bei vorwiegend starker Eiterabsonderung, zumal wein abgestossene kleine Fetzell von Granulationsgewebe vermuthen lassen, dass sich auf der Blasenschleimhaut ulcerirende Partien befinden. Unter solchen Verhältnissen wirken diese Injectionen auf die Blasenschleimhaut nicht anders, wie auf wunde Flächen, in kleinen Dosen adstring irend, in grösseren kauterisirend, - und zwar das Chlorzink stärker wie das Arg. nitricum. Ob man mit verdünnten Chlorzinklösungen auch auf das Sediment und den Katarrl einwirken kann, dafür gebelı die wenigen

') ef. W. Kühne, Lehrbuch der physiologischen Chemie, pag. 274 und pag. 401 . 
Versuche, walche wir in dieser Richtung angestellt haben, noch zu wenig Anhaltspankte.

Resimiren wir zum Schluss die Ergebnisse meiner oben niedergelegten trörterungen über die differentielle Indication der einzelnen Jocal anzuwendenden Mittel, so darf man sie etwa folgendermaassen präcisiren:

Während die Carbolsäure und das Kali hypermanganicum sich besonders bei Blasenkatarhen -mit starker Zersetzung eignen, empfiehlt sich die Salicylsäure speciell bei vorwiegend eiterigen Katarrhen. Bagegen sind beim Vorhandensein dicker zähschleimiger, massiger Niederschläge die Kochsalzausspillungen - wie überhaupt solche von alkalisehen Lösungen - vorutiehen. Bei andauernder profuser Eiterabsonderung sind sehr zweckentsprechend intercurrente Injectionen von einprocentigen Argentumnitricumlösungen oder zweiprocentigen Chlorzinklösungen zu machen. Bei der Blasendiphtheritis dagegen sind stärkere Carboleinspritzungen von unubertroffener Wirksamkeit. - 